\title{
Eye Movement Control During Reading: Foveal Load and Parafoveal Processing
}

\author{
W. Schroyens \\ University of Leuven, Leuven, Belgium \\ F. Vitu \\ CNRS, Université René Descartes (Paris V), Paris, France \\ M. Brysbaert \\ University of Gent, Gent, Belgium \\ G. d'Ydewalle \\ University of Leuven, Leuven, Belgium
}

We tested theories of eye movement control in reading by looking at parafoveal processing. According to attention-processing theories, attention shifts towards word $n+1$ only when processing of the fixated word $n$ is finished, so that attended parafoveal processing does not start until the programming of the saccade programming to word $n+l$ is initiated (Henderson \& Ferreira, 1990; Morrison, 1984), or even later when the processing of word $n$ takes too long (Henderson \& Ferreira, 1990). Parafoveal preview benefit should be constant whatever the foveal processing load (Morrison, 1984), or should decrease when processing word $n$ outlasts an eye movement programming deadline (Henderson \& Ferreira, 1990). By manipulating the frequency and length of the foveal word $n$ and the visibility of the parafoveal word $n+1$, we replicated the finding that the parafoveal preview benefit is smaller with a low-frequency word in foveal vision. Detailed analyses, however, showed that the eye movement programming deadline hypothesis could not account for this finding which was due not to cases where the low-frequency words $n$ had received a long fixation, but to cases of a short fixations less than $240 \mathrm{msec}$. In addition, there was a spill-over effect of word $n$ to word $n+1$, and there was an element of parallel processing of both words. The results are more in line with parallel

Requests for reprints should be sent to Dr. Walter Schroyens, Laboratory of Experimental Psychology, University of Leuven, Tiensestraat 102, B-3000 Leuven, Belgium. E-mail: Walter.Schroyens@psy.kuleuven.ac.be

The research was partially founded by the TOURNESOL project between the University of Leuven (G. d'Ydewalle, M. Brysbaert), and the Université René Descartes (F. Vitu; associated with the CNRS); as well as a 4-month research fund of the ERASMUS exchange programme and a 3-month research fund of the Cultural and Scientific Service of the French Embassy in Belgium for W. Schroyens (currently a junior research fellow of the Flanders Fund for Scientific Research, FWO Vlaanderen, Belgium). We would also like to acknowledge Fredérique Lavigne-Tomps and Jeremy Pacht for their effort in stimulating discussions, and John Driver, as well as the anonymous referees, for their helpful critiques on previous drafts of the manuscript. 
processing limited by the extent to which the parafoveal word processing on fixation $n$ can be combined with the foveal word processing on fixation $n+1$.

During reading, the eyes move across the lines of text by making saccades of variable size. These saccades are meant to bring new information into the centre of the visual field (foveal vision). The amount of information that can be extracted during the subsequent fixation is strongly constrained by visual acuity (or the fact that the visibility of a letter decreases with its distance from the fixation point; Hirsch \& Curcio, 1989) and by lateral masking (Bouma, 1978). As shown in several studies, the probability of correctly identifying an isolated word drops dramatically the further away the eyes are from the centre of the word (Bouma, 1978; Brysbaert, Vitu, \& Schroyens, 1996; McConkie, Kerr, Reddix, Zola, \& Jacobs, 1989; Nazir, O'Regan, \& Jacobs, 1991). On the other hand, there is also considerable evidence that, in addition to foveal word processing, parafoveal and peripheral information is extracted and used in the process of reading. Studies with the moving-window technique have shown that semantic information is extracted up to 6-8 characters to the right of the fixation location, and that more global visual information related to word length can be extracted up to 15 characters to the right of the fixation location (McConkie \& Rayner, 1975). Several other studies have shown that the processing of a word is facilitated when the word was available in parafoveal vision during the previous fixation. In particular, the time that the eyes spend on a word (the gaze duration) is shorter when the word was visible in parafoveal vision than when it was masked (see e.g. Balota \& Rayner, 1991; Inhoff, 1989; Inhoff \& Rayner, 1980, 1986; Rayner, McConkie, \& Zola, 1980; Rayner, Well, Pollatsek, \& Bartera, 1982; Vitu, 1991).

At present, although it is clear that both foveal and parafoveal information is extracted during a fixation, little is known about the way in which these two forms of information are handled in the time course of an eye fixation. The question particularly applies to the case of text reading where two different words are presented in foveal and parafoveal vision. Although the eyes sometimes land on the space between two words, the most common observation is that the eyes initially land between the beginning and the middle of a word (Dunn-Rankin, 1978; Rayner, 1979; Vitu, O’Regan, \& Mittau, 1990; Vitu, McConkie, Kerr, \& O'Regan, 1999). On these occasions, does processing of the foveal and the parafoveal word occur in parallel or does foveal processing precede parafoveal processing due to a selective-attention mechanism?

Among theories of eye guidance in reading, several proposals have been made concerning the time course of parafoveal processing in relation to foveal processing (Henderson \& Ferreira, 1990; Just \& Carpenter, 1980; McConkie, 1979; Morrison, 1984; Pollatsek \& Rayner, 1990; Rayner \& Pollatsek, 1989). The most commonly accepted view relies on the idea that the selection of visual information is necessary prior to its subsequent processing (cf. the early-selection models in visual perception; McCann, Folk, \& Johnston, 1992; Mozer \& Behrmann, 1991). This view holds that in reading the foveal and the parafoveal words are processed serially during the time period of a fixation. As originally proposed by Morrison (1984), the sequence of events within a fixation would be organized as follows. When the eyes land on a word, attention is usually focused on that word. This makes it possible to process the information of the foveal word $n$ selectively and to ignore the information from the parafoveal word $n+1$. Only when word $n$ has been 
identified does attention shift to the next word, and the processing of the parafoveal word $n+1$ is initiated. As a shift of attention is assumed to be followed systematically by an eye movement, a saccade towards word $n+1$ will be programmed, and the processing of word $n+1$ in parafoveal vision will continue until the saccade is executed. The time spent on attentional processing of the parafoveal word, therefore, is quite constant, depending only on minor fluctuations associated with saccade programming. Consequently, the amount of information that can be extracted from the parafoveal word should depend only on the visual and linguistic characteristics of this parafoveal word and not on the difficulty of the foveal word.

According to Henderson and Ferreira (1990), there may be some instances in which the programming of a saccade is initiated without a prior shift of attention. This is the case when the processing of the foveal word takes longer than a hypothetical eye movement programming deadline, which prevents the eyes from staying too long on the same location without moving. Then, a refixation of the foveal word is programmed, as this word is still at the focus of attention. During this programming period, however, it is possible that the processing of the foveal word terminates and that attention shifts towards word $n+1$. On those particular instances, the refixation of the foveal word will be cancelled and the saccade parameters quickly adjusted in order to bring the eyes on the next word. This will reduce parafoveal preprocessing to the saccadic programming time that remains after the cancelling.

According to this saccade programming deadline hypothesis, the time devoted to parafoveal word processing depends not only on random variations of the saccadic programming time but also on the foveal processing load. The more difficult the processing of the fixated word, the higher the chances that a forward saccade is a cancelled refixation, which reduces the amount of information that can be extracted from the parafovea.

Both Morrison's (1984) and Henderson and Ferreira's (1990) theories can be contrasted with a more intuitive hypothesis based on an extension of the so-called lateselection models of visual perception. This consists of the assumption that foveal and parafoveal information are processed in parallel. According to this view, processing of a word in the parafovea is not delayed until the foveal word has been identified but is initiated as soon as the eyes land close enough to the word so that some visual information can be extracted. It therefore usually starts when the eyes land on the previous word $n$ and lasts as long as this word is fixated. As the time spent on a word is related to the difficulty of that word, the amount of parafoveal processing should be a function of the foveal processing load: The more difficult the processing of word $n$, the greater the duration of parafoveal processing and the greater the amount of parafoveal information collected. This prediction is the opposite of that made by Henderson and Ferreira (1990).

It should be noted that a parallel model of foveal and parafoveal word recognition need not necessarily predict a positive relation between parafoveal preprocessing and time spent on the foveal word. A parallel model that does not make this prediction was proposed by Schiepers (1980). Starting from the finding that it takes an average $90 \mathrm{msec}$ per degree of eccentricity longer to identify a word (see also Rayner \& Morrison, 1981), Schiepers hypothesized that the parafoveal word would be processed in parallel with the foveal word, but with a delay of about $200 \mathrm{msec}$ (because it is typically situated at an eccentricity of $2^{\circ}$ ). This initial parafoveal processing is added to the foveal processing 
during the next fixation, at which time it can reinforce word recognition if both sources of information activate the lexicon in synchrony. In this respect, Schiepers notes that it is probably not a coincidence that "in reading, the saccade lengths are about $2^{\circ}$ and fixations last about $200 \mathrm{~ms}$ " (p. 79), so that "On average, the visual information from a word at one saccade length to the right of the fovea arrives simultaneously with the information from the next eye fixation in which that word is in the fovea" (p. 78). This combination of information would help to speed up word recognition. Interestingly, Schiepers (1980) further notes that his model (like that of Henderson and Ferreira, 1990, one decade later) predicts that "Lengthening of the fixation duration means slowing down recognition [of the parafoveal word], for the additive component of the following fixation is delayed" (p. 79).

Two recent studies (Henderson \& Ferreira, 1990; Kennis on \& Clifton, 1995) investigated the question of whether parafoveal processing depends on foveal processing load, and the results appeared to favour the serial attentional process with saccadic deadline proposed by Henderson and Ferreira (1990) (or the parallel view defended by Schiepers, 1980 , although this was not mentioned). The parafoveal preview benefit of seeing the target word in parafoveal vision on an earlier fixation decreased with the difficulty of the foveal word (called the boundary word). It was smaller for low-frequency than for highfrequency boundary words (Henderson \& Ferreira, 1990), and the cost was independent of the frequency of the target word (Kennison \& Clifton, 1995). It was also smaller for boundary words that could not be predicted from the preceding syntactic information (Henderson \& Ferreira, 1990, Experiment 2).

However, the above results are not necessarily supportive of Henderson and Ferreira's (1990) hypothesis. First, as noted by Kennison and Clifton (1995), the difference in preview benefit between high- and low-frequency boundary words was limited to those cases in which the eyes were located on the last three characters of the boundary word. Second, if the reduced preview benefit were due to a cancelled refixation, as assumed by Henderson and Ferreira (1990), then the reduction in parafoveal preview benefit with low-frequency boundary words should be observed only for those instances in which the boundary word was seen in a single fixation. When a boundary word receives two fixations, this is because processing was not terminated before the occurrence of the refixation saccade. In these particular cases, word $n$ is very likely to be identified during the second fixation and before a new saccade is being programmed (i.e. like the case of a real single fixation, without a cancelled refixation).

Unfortunately, so far neither of the studies reporting an interaction between foveal load and preview benefit has made a distinction between single and double fixations on the boundary word (Henderson \& Ferreira, 1990; Kennison \& Clifton, 1995). A close examination of both sets of data, however, suggests a considerable proportion of doublefixation cases in both studies, particularly for low-frequency boundary words. Kennison and Clifton (1995) report an average number of fixations of 1.26 for low-frequency boundary words and 1.19 for high-frequency boundary words. Henderson and Ferreira (1990) report gaze durations that were systematically longer than first fixation durations (the difference being largest for low-frequency unpredictable boundary words). Therefore, it is possible that the reduced parafoveal preview benefit for low-frequency boundary words was due to a higher proportion of two-fixation cases, which would be inconsistent 
with Henderson and Ferreira's (1990) hypothesis. On the other hand, a pure parallel processing hypothesis, which asserts that processing of the parafoveal word starts as soon as the eyes are close enough to that word, could account for this finding. On those instances where the boundary word is fixated with two consecutive fixations, it is reasonable to assume that parafoveal processing is more likely to occur at the beginning of the second fixation, because, as indicated by several reading studies, this fixation is likely to be closest to the word's end and thus closer to the next word (Vitu, 1990; Vitu et al., 1999). As second-fixation durations are generally shorter than single-fixation durations, less parafoveal processing benefit would accrue in the two-fixation cases (i.e. when the boundary word was of low frequency), simply because of their shorter second fixation near the boundary word's end. Thus, it is conceivable that the differences in parafoveal preview benefit previously observed are not due to passing of the suggested eye movement programming deadline but are simply an artefact of the way the boundary word was explored.

Furthermore, if, as assumed by Henderson and Ferreira (1990), foveal-on-parafoveal effects are the result of the existence of two different populations of single fixations (real single fixations vs. cancelled refixations), then the distribution of single fixations should be bimodal. However, as shown in several studies, this is not the case: The distribution of single-fixation durations is unimodal with no apparent fixation duration cut-off (Kennison \& Clifton, 1995; Vitu \& O'Regan, 1995). Furthermore, if refixations are due to exceeding the eye movement programming deadline, single fixations should be shorter than the first fixations of the two-fixation cases. Again, recent data point to the opposite pattern: Single-fixation durations are systematically longer than the durations of first fixations in the two-fixation cases (Rayner, Sereno, \& Raney, 1996; Vitu \& O'Regan, 1995; see also Vitu et al., 1999). These two findings question the validity of the eye movement programming deadline hypothesis but are not sufficient to rule out the possibility that a serial attentional mechanism determines the time course of foveal and parafoveal word processing.

In order to assess better the role of attention in the time course of foveal and parafoveal word processing, we tested the influence of foveal processing load on the parafoveal preview benefit. This study follows up the studies of Henderson and Ferreira (1990) and Kennison and Clifton (1995), but in a better controlled and simpler paradigm where subjects' eye movements were recorded while they were reading three isolated words presented simultaneously: a boundary stimulus initially presented in foveal vision, a target word in the parafovea, and a third word to avoid any "wrap up" processes on the target word. In order to manipulate the foveal load, we varied both the length ( 3 or 5 letters) and the frequency of the boundary stimulus; it was a high-frequency word, a low-frequency word (as in the previous studies), or a meaningless z-letter string, which served as a control condition. Furthermore, to see whether parafoveal processing changed as a function of target word characteristics, the frequency of the target word was also manipulated (high vs. low). This word was either visible or masked when presented in parafoveal vision. The parafoveal preview benefit was measured by comparing the eye movement pattern on the target word in the visible and in the masked preview conditions.

Because predictions from both serial attentional and parallel processing hypotheses are clearer for cases where the boundary stimulus is fixated in a single fixation (see earlier), we 
maximized the probability of such instances by using short boundary stimuli (3 to 5 letters) and by presenting the boundary stimulus centred on the fixation location. As shown in several studies, the probability of refixating a word or a z-letter string is much smaller for short stimuli initially fixated at their centre (see e.g. O'Regan \& Lévy-Schoen, 1987; O’Regan, Lévy-Schoen, Pynte, \& Brugaillère, 1984; Vitu, O’Regan, Inhoff, \& Topolski, 1995; Vitu, O'Regan, \& Mittau, 1990). In addition, analysis of parafoveal preview effects was restricted to those cases in which the boundary stimulus had been fixated with a single fixation. Controlling the boundary word length and the initial fixation position also made it possible to ensure that the first letters of the parafoveal word were located within the perceptual span (Brysbaert et al., 1996; McConkie \& Rayner, 1975).

According to serial attention theories, parafoveal processing begins only when the saccade to the next word starts being programmed (Henderson \& Ferreira, 1990; Morrison, 1984), or even later in the case of a cancelled refixation (Henderson \& Ferreira, 1990). Hence, the parafoveal preview benefit should be independent of the foveal processing load (Morrison, 1984), or should decrease only for those cases where the boundary word takes longer to process than the eye movement programming deadline (Henderson \& Ferreira, 1990). A pure parallel-processing hypothesis, on the other hand, predicts that more information can be extracted from a parafoveal target word when the eyes stay longer on the foveal boundary word. Both theories also differ with respect to the effect of the parafoveal target word frequency. Although both the serial attentional and the parallel hypotheses predict larger preview benefits for high-frequency target mords, the hypothesis proposed by Henderson and Ferreira (1990) cannot predict differential effects of foveal load on the preview benefit for different parafoveal target word frequencies. This is because the differential preview effect is assumed to result only from the efficiency of processing the foveal word with respect to the eye movement programming deadline.

\section{EXPERIMENT}

\section{Method}

\section{Subjects}

A total of 28 subjects participated in the experiment. They were first- and fifth-year psychology students, or were otherwise associated with the Laboratory of Experimental Psychology of the University of Leuven. All subjects had normal or corrected-to-normal vision and were native Dutch speakers.

\section{Design}

Subjects served as their own controls in a 2 (boundary word length: three- or five-letter words) by 3 (boundary word frequency: low-frequency, high-frequency, or z-strings) by 2 (target word frequency: low- or high-frequency) by 2 (parafoveal preview condition of the target words: masked or visible) repeated measures design. The items were presented in a different random order for each subject. Four counterbalanced lists were used in the different conditions. 


\section{Materials}

We constructed a list of 540 word triads: 360 experimental trials and 180 filler items. An additional list of 60 word triads served as practice items. The first two words of the triad were the stimuli of interest; the third word was merely added to elicit normal reading behaviour on the second word. The first word (called the boundary word) of the experimental items was either three or five letters long. This boundary word was of low frequency, high frequency, or was a homogeneous z-string of three or five letters. The second word (called the target word) was a low-frequency or a high-frequency word of seven letters in the experimental trials. The list of experimental items was divided in to 24 subsets with 15 items each, to get a full matrix of the 2 (boundary word length) $\times 3$ (boundary word frequency) $\times 2$ (target word frequency) $\times 2$ (parafoveal preview condition) design. The frequency of the boundary and the target word were matched for the two lengths of the boundary word. Mean frequencies per million for the low-frequency three-letter, low-frequency five-letter, high-frequency three-letter, and high-frequency five-letter boundary words were $0.9,0.7,64.5$, and 108.7, respectively. For the seven-letter target words these were 0.6 (low frequency) and 69.3 (high frequency) (see the Appendix for further details). From this base list, four counterbalanced lists were constructed by crossing the subsets for the masked and the visible preview condition, and by crossing the target words over the lengths of the boundary words.

To reduce the amount of lateral masking and to control for potential orthographic overlap, the boundary words were paired with their target words under the constraint that the initial letter of both words had to be different. In addition, we ensured that no semantic link existed between the pairs of boundary and target words. The third word was added to elicit a more natural reading situation for the target word. The length of the third word was between four and eight characters.

A set of 180 filler items was added to the list of experimental trials, in order to create sufficient variability in the length of the boundary and the target words and to reduce the probability that subjects developed specific scanning strategies when reading the experimental trials. The same parafoveal preview conditions were used for the filler items in order to remain compatible with the experimental trials. The length of the boundary words in the filler items was either four or six letters $(90$ cases each). To match the proportion of $z$-strings in the experimental boundary words, one-third of the boundary words in the filler items were z-strings (30 cases for both four- and six-letter z-strings). These filler items were randomly interspersed among the experimental items. The word frequency of the filler items was situated at a level intermediate between the high and low frequencies of the experimental items. As with the experimental items, we paired boundary and target words with a different initial letter and minimized semantic linkages between them.

\section{Procedure}

When subjects arrived in the laboratory, a bite bar was prepared for them to minimize head movements. If they were not acquainted with the eye-tracking system, they were familiarized with the system, and the calibration procedure was explained to them. The calibration itself took 5 to $15 \mathrm{~min}$. The distance between the eyes and the screen was $124 \mathrm{~cm}$, such that three character spaces equalled $1^{\circ}$ of visual angle. We used 15 fixation points to calibrate the eye-tracking system ( 5 points on both diagonal axes, and 5 points iteratively below and above the central horizontal axes of the screen).

We asked the subjects to read the words as they would read them normally. On each trial subjects first had to fixate a blank space between two vertical lines that were placed one above the other halfway between the left border and the centre of the screen. When the eye tracker detected a $60-\mathrm{msec}$ fixation in the fixation region of half a character to both sides of the exact fixation 
location (letters were made by using a $16 \times 16$ matrix in graphics mode of Turbo Pascal 4.0), the fixation point disappeared, and the word triad was presented with the boundary word centred on the fixation point. When the eyes crossed the boundary situated at one third of the space between the boundary word and the target word, the boundary word was masked and the target word became visible if it had been masked before. The blank space between the boundary words and the target word was one and a half characters (24 pixels). Parafoveal masking of the words was accomplished by randomly scrambling the pixels that formed the letters of the words, so that the overall luminance of the parafoveal preview was the same in the masked and the unmasked conditions. The third word was always masked at the beginning of a trial. When the boundary between the target word and the third word was crossed (again situated at one third of the blank space), the target word was masked and the third word became visible. The word triad was removed from the screen as soon as the subject looked at a fixation cross that was displayed seven character spaces to the right of the third word.

After a word triad was removed from the screen, a question mark was presented at the bottom left side of the screen. Subjects had to indicate whether or not one of the words in the triad referred to an article of clothing by pressing a button with either the right hand ("no") or the left hand (“yes"). Subjects were encouraged to perform as well as possible and were informed that in only a few trials $(6.1 \%)$ would one of the words refer to clothes. These trials were all filler items. We told the subjects that sometimes the first word would be a z-string, which obviously did not refer to clothing. As soon as the subjects pressed the button, feedback about the correctness of the response was given by presenting either the letter " $F$ " for an error or " $C$ " for a correct answer. The simplicity of the task resulted in an accuracy level of $99.5 \%$ correct answers on the experimental items. The feedback was presented for half a second, after which the screen was blanked and the next trial was started.

Calibration was checked at the beginning of each of the nine blocks of 60 trials or when the subject had problems initiating a trial. The calibration was checked by presenting five fixation crosses from left to right on the same horizontal axis as that on which the stimuli were presented. Pressing the left button produced a marker that showed the position at which the subject should be looking according to the tracker. If any deviation between the fixation cross and the marker could not be resolved by the subjects resuming their original position or adjusting the zero-point of the eye-tracking system, the procedure was interrupted and the eye tracker was recalibrated. An interruption of the procedure to recalibrate the eye-tracking system during a block of trials led to a reiteration of the interrupted block. A reiteration of trials occurred twice: For two subjects the procedure was interrupted after the eleventh and the fourth trial, respectively. Those trials that were presented twice were excluded from the analyses.

\section{Apparatus}

Eye movements were tracked with a generation-V Stanford Research Institute Dual Purkinje eye tracker that has a spatial accuracy of $1 \mathrm{~min}$ of arc. Eye movements were recorded from the right eye, but vision was binocular. The eye tracker was interfaced with two IBM-compatible microcomputers. The first computer recorded the eye movement parameters. Horizontal and vertical eye positions were sampled ever $1 \mathrm{msec}$. The eye position parameters were continuously sent to the second computer, which was used to control the stimulus presentation in graphics mode on a 13" CRT monitor with $60-\mathrm{Hz}$ refresh rate. The first computer was interfaced with two response buttons. The decision to change the stimulus display was contingent on the position of the eyes (see earlier). The decision to start the next trial was based on the signal of the button press, which indicated the decision made by the subject. 


\section{Result}

Analyses of variance (ANOVAs) were run on the means per subject per condition and the means per item. These means excluded a number of trials. First, there were "noise trials" with key-press failures, track losses, or blinks on the boundary or target word, or on which the word stimuli were not presented in synchrony with the eye movements. Second, trials for which fixation durations, after logarithmic transformation, were longer than the mean plus two and a half times the standard deviation or were shorter than the mean minus two and a half standard deviations, were also excluded. Finally, trials in which an intra-word refixation occurred on the boundary words were discarded. The percentages of trials remaining after these successive eliminations were $87 \%, 82 \%$, and $80 \%$. Thus, for the ANOVA, the means used for each subject in each condition were based on about 12 items. The excluded trials were equally distributed across conditions.

To measure the parafoveal preview benefit in the different experimental conditions, we compared the eye movement data for the target word in the visible and the masked preview conditions. Several measures were used: (1) the gaze duration (i.e. the total time that the eyes spent on the target word), (2) the refixation probability, (3) the duration of single fixations (i.e. gaze durations on target words that were processed with only one fixation), and (4) the first-fixation duration (i.e. the duration of the initial fixation independent of the number of subsequent fixations on that word).

\section{Boundary Word Single Fixation Durations}

Before testing whether the complexity of the boundary stimulus affected the amount of information extracted in parafoveal vision, we ran several control analyses. In order to ensure that our manipulation of foveal processing load was successful, we checked whether subjects looked longer at boundary words that were assumed to be difficult to process than at boundary words that were thought to be easy. As only cases with one fixation on the boundary word were taken into account (see earlier), this analysis consisted of comparing average single-fixation durations. Results are presented in Figure 1. As expected, reading times were longer for five-letter than for three-letter boundary stimuli, $F_{1}(2,54)=31.52, p<.0005, F_{2}(2,696)=256.16, p<.0005$. They were also longer for words than for z-letter strings, and longer for low-frequency than for high-frequency words. Indeed, there was a significant effect of the boundary stimulus type, $F_{1}(2,54)=21.39, p<.0005, F_{2}(2,696)=142.28, p<.0005$, and this was true for both three-letter and five-letter boundary stimuli, $F_{1}(2,54)=15.61, p<$ $.0005, F_{2}(1,696)=56.66, p<.0005$ and $F_{1}(2,54)=22.15, p<.0005, F_{2}(1,696)=$ $91.48, p<.0005$, respectively.

It should be noted, however, that although the main effects were in the expected direction, the word frequency effect was clearer for the five-letter boundary words than for the three-letter boundary words. This led to a significant interaction between boundary stimulus type and boundary word length, $F_{1}(2,54)=4.97, p<.05, F_{2}(2,696)$ $=5.86, p<.05$. No other main effect or interaction was significant. In addition, further analyses showed that the reading times of the boundary stimulus were not affected by the 


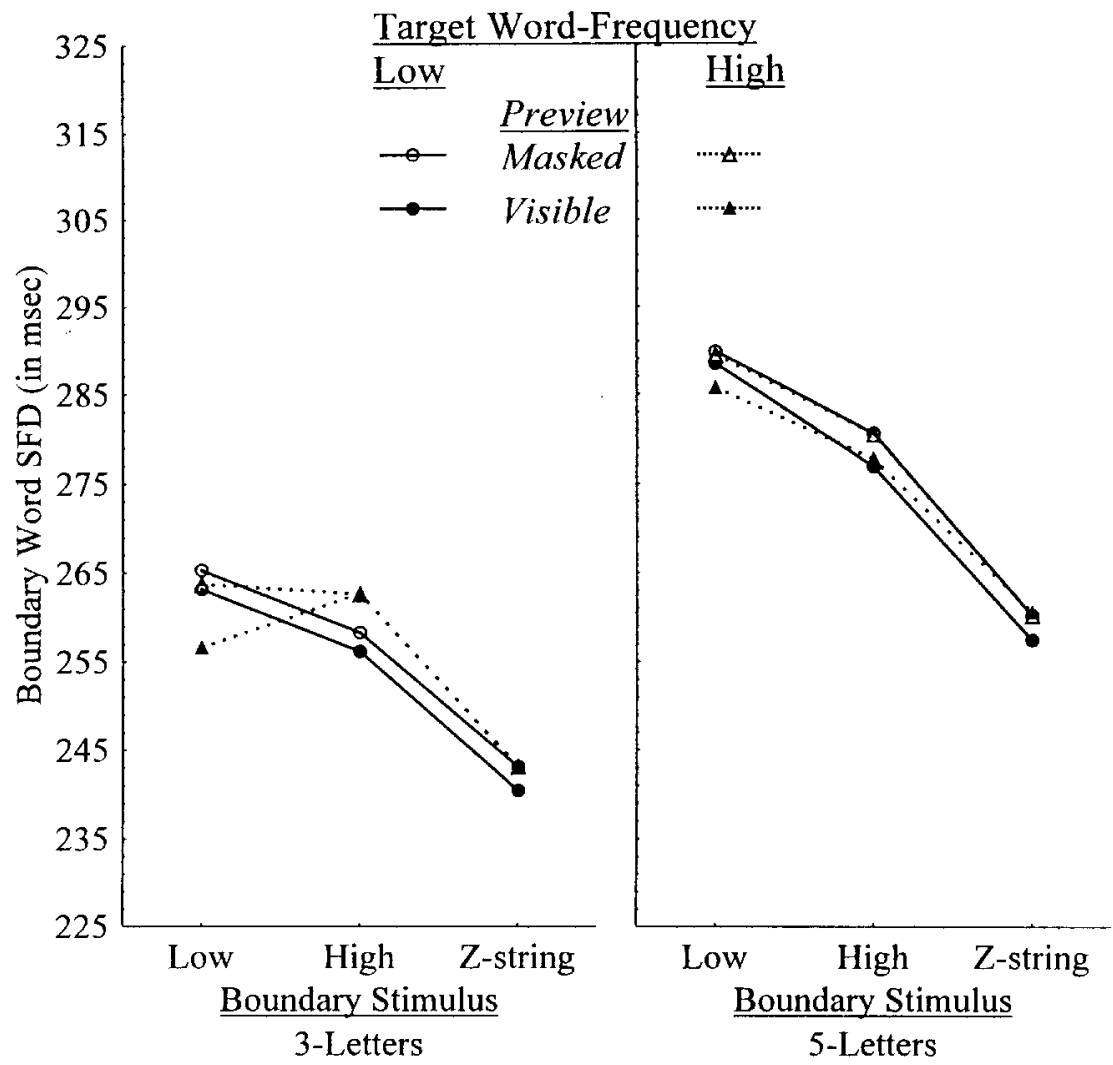

FIG. 1. Mean single-fixation durations (SFD, in $\mathrm{msec}$ ) on the boundary stimuli as a function of their type and length, and the frequency and preview condition of the target words.

characteristics of the second word (the target word), such as its frequency, $F \mathrm{~s}<1$, or parafoveal visibility, $F_{1}(1,27)=2.41, p>.10, F_{2}(1,696)=2.81, p>.09$. Several other control analyses were run, which consisted in testing whether the eye's position in the boundary word (from which the eyes were launched) and the eye's initial landing position varied between the different experimental conditions. ${ }^{1}$

\footnotetext{
${ }^{1}$ As shown in several studies, the eye's location in a word strongly affects how many letters can be extracted from both the fixated word and the next parafoveal word (Brysbaert, Vitu, \& Schroyens, 1996; McConkie, Kerr, Reddix, Zola, \& Jacobs, 1989; Nazir, O'Regan, \& Jacobs, 1991). When we were testing for parafoveal preview effects, it was therefore necessary to ensure that the eyes were launched from the same location and landed on average on the same location in the different experimental conditions. In the present experiment, major differences in launch site were not expected as the eye tracker checked for an adequate fixation at the beginning of each trial (see the Procedure section). However, because an uncertainty region had been defined for the checking, it still seemed safer for us to look for possible effects. No main or interaction effects were significant for the launch sites. For the landing position there was a small but reliable effect of the boundary stimulus, $F_{1}(2,54)=$ $6.929, p=.005, F_{2}(2,696)=22.89, p<.0005$ : The landing position was 0.2 character positions more to the right after a boundary z-string (3.76) than after a high-frequency (3.54) or low-frequency word (3.50).
} 


\section{Target Word Gaze Durations}

In order to test the effects of parafoveal preview, we first looked at the gaze durations on the target word as a function of the type of boundary stimulus and the visibility of the target word. As can be seen in Figure 2, there was a clear advantage when the target word was visible in parafoveal vision compared with when it was masked $(285 \mathrm{msec}$ vs. $297 \mathrm{msec}), F_{1}(1,27)=31.44, p<.0005, F_{2}(2,696)=32.69, p<.0005$. This preview benefit was larger for high-frequency target words $(16 \mathrm{msec})$ than for low-frequency target words $(8 \mathrm{msec})$, resulting in a significant interaction between visibility of the target word and frequency of the target word, $F_{1}(1,27)=8.41, p<.01, F_{2}(1,696)=1.31, p>.20$.

In apparent support of Henderson and Ferreira's (1990) hypothesis, the preview benefit was smaller for low-frequency boundary words than for high-frequency boundary words or z-letter strings. The interaction between the preview condition and the boundary stimulus type was significant, $F_{1}(2,54)=5.61, p<.01, F_{2}(2,696)=3.57, p<.05$. The parafoveal preview benefit was significant for both high-frequency boundary words and z-letter strings, (respectively, $301 \mathrm{msec}$ vs. $286 \mathrm{msec}$, and $282 \mathrm{msec}$ vs. $265 \mathrm{msec}$ ) $F_{1}(1,27)=$ $18.29, p<.0005, F_{2}(1,696)=17.03, p<.0005 ;$ and $F_{1}(1,27)=37.81, p<.0005$, $F_{2}(1,696)=21.50, p<.0005$, but not for low-frequency boundary words $(308 \mathrm{msec}$ vs.

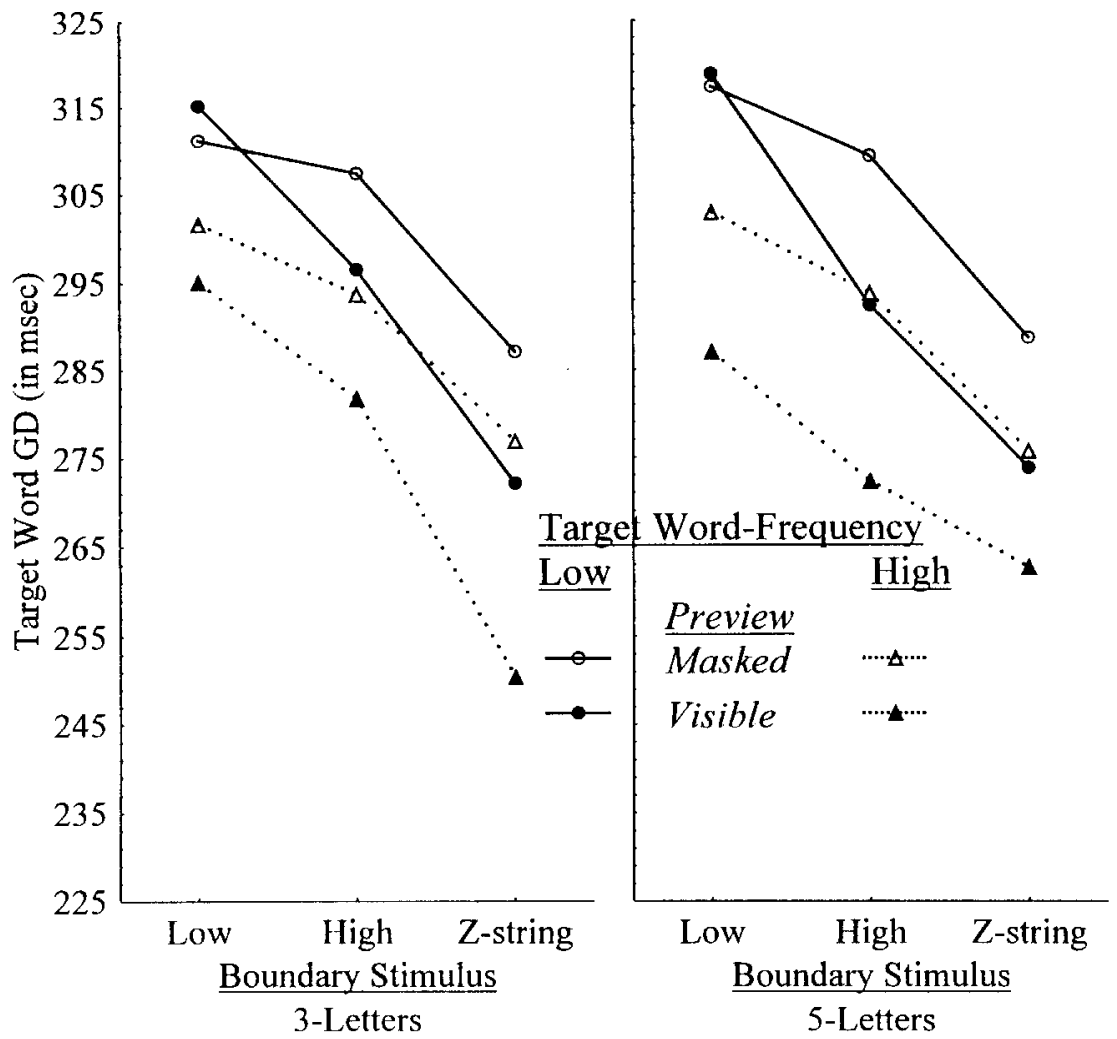

FIG. 2. Mean gaze durations (GD, in $\mathrm{msec}$ ) on the target words as a function of their frequency and preview condition, and the type and length of the boundary stimuli. 
$304 \mathrm{msec}), F_{1}(1,27)=1.56, p>.20, F_{2}(1,696)=1.30, p<.0005$. Although the three-way interaction between parafoveal preview, boundary stimulus type, and target word frequency was not significant, $F \mathrm{~s}<1.5$, specific comparisons showed that the observed decrease of the preview benefit with the complexity of the boundary stimulus was clearly present only for low-frequency target words. The interaction between parafoveal preview and boundary stimulus complexity was significant for low-frequency target words, $F_{1}(2,54)=$ $7.61, p<.005, F_{2}(2,696)=4.66, p<.01$, but not for high-frequency target words, $F \mathrm{~s}<1$. For high-frequency target words, there was just a tendency for a decrease with three-letter boundary stimuli, and no decrease at all with five-letter boundary stimuli.

In addition, it is interesting to note a significant effect of boundary stimulus type in both the visible, $F_{1}(2,54)=39.49, p<.0005, F_{2}(2,696)=55.80, p<.0005$, and the masked preview condition, $F_{1}(2,54)=19.88, p<.0005, F_{2}(2,696)=29.92, p<.0005$. Overall, gaze durations on the target word were shorter after a boundary z-string $(273 \mathrm{msec})$ than after a high-frequency boundary word $(293 \mathrm{msec})$ or a low-frequency boundary word $(306 \mathrm{msec})$. This indicates a spill-over effect of the boundary stimulus. Such a spill-over effect of the boundary stimulus on the target stimulus was also found by Kennison and Clifton (1995), who used a situation more like natural reading of text.

Thus, the results for the gaze duration measure seem to replicate the previous findings of Henderson and Ferreira (1990), and Kennison and Clifton (1995), which showed smaller preview benefits for low- than for high-frequency boundary words. An additional finding of the present experiment is that the effect remains when the analysis is restricted to instances in which the boundary word was seen in a single fixation (see the Introduction). It may also be noted that the difference in parafoveal preview benefit from low- and high-frequency boundary words was not replicated for the difference between highfrequency words and $z$-strings, despite the fact that the $z$-strings were considerably easier to process. If the analysis was restricted to these two conditions, there was no significant interaction between the preview condition and the type of boundary stimulus, $F \mathrm{~s}<1$.

\section{Target Word Refixation Probability}

As shown in several studies, the gaze duration on a word is a composite measure that depends on both the number of fixations on the word and the duration of the individual fixations (Vitu et al., 1999). To understand better the pattern of results obtained with the gaze duration measure, we therefore tested the influence of the boundary stimulus type on both the probability of refixating the target word (or likelihood of making more than one fixation on the target word), and the duration of single fixations (i.e. cases where the initial fixation on the target word was not followed by an additional fixation). The analysis of first-and second-fixation durations in the two-fixation cases is not presented here as there were not enough two-fixation cases.

As can be seen in Figure 3, the pattern of results obtained with the target word refixation probability measure is not as clear as that for gaze duration. There was no effect of the parafoveal preview condition $(16.6 \%$ vs. $15.8 \%), F \mathrm{~s}<1.5$, nor was there an overall interaction between parafoveal preview condition and boundary stimulus type, $F_{1}(2,54)=1.94, p>.15, F_{2}(2,696)=1.10, p>.15$. However, when we restricted the analysis to conditions with low- and high-frequency boundary words, a tendency for an 


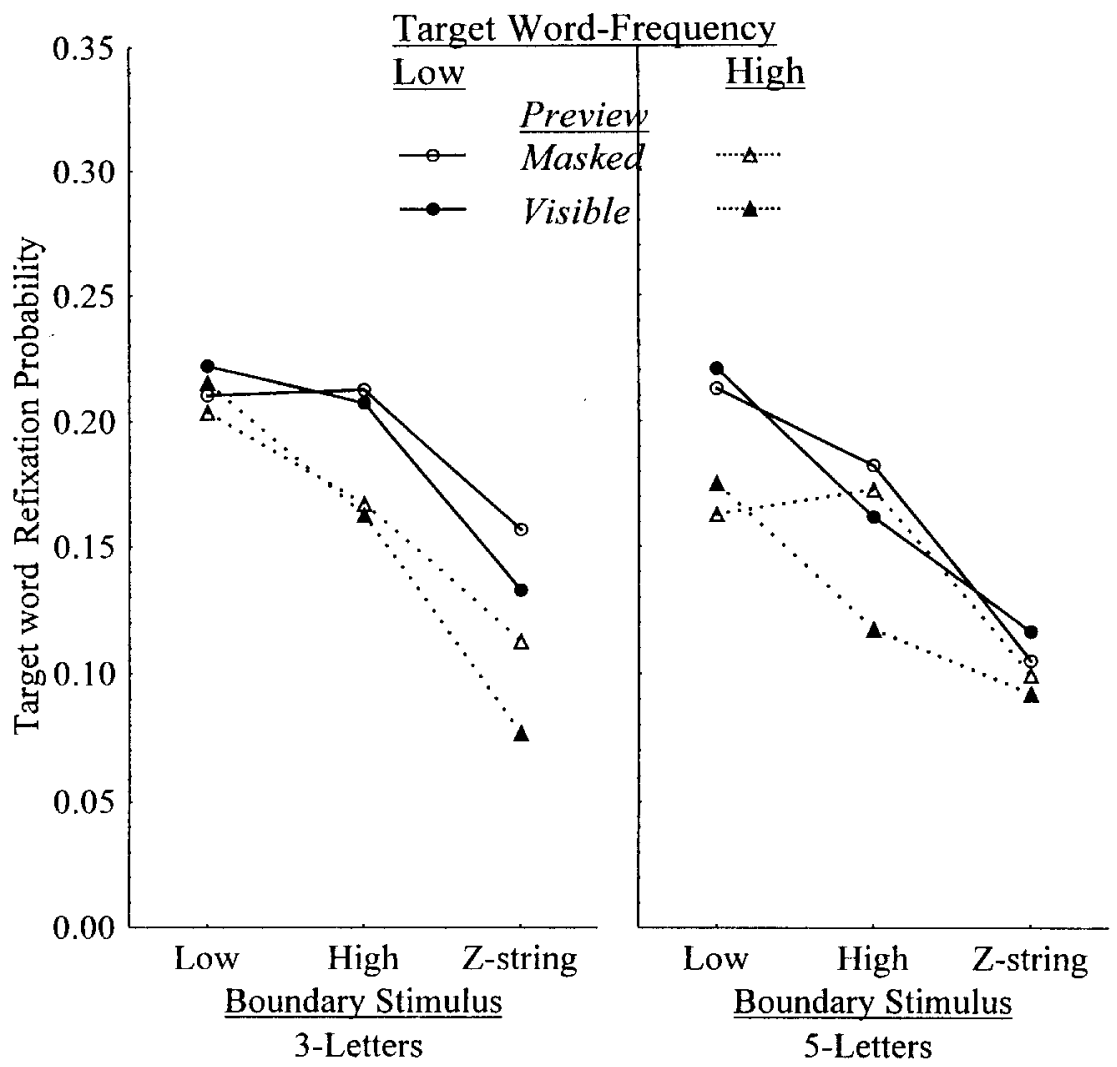

FIG. 3. Mean refixation probabilities on the target words as a function of their frequency and preview condition, and the type and length of the boundary stimuli.

interaction with parafoveal preview condition emerged (masked: $19.7 \%$ vs. $18.4 \%$; visible: $20.8 \%$ vs. $16.2 \%), F_{1}(1,27)=3.65, p<.07, F_{2}(1,696)=2.13, p>.30$.

\section{Target Word Single-fixation Durations}

The analysis of single-fixation durations on the target word (see Figure 4) was based on $63.7 \%$ of the data only, or an average of 9.5 observations per subject per condition (or 4.8 per item), because excluding refixation cases entailed a further reduction in the data. Missing data from two subjects who refixated all target words in a given condition were replaced by the average mean single fixation duration in that condition over the other subjects. ${ }^{2}$

\footnotetext{
${ }^{2}$ By removing target word refixation cases to calculate single-fixation durations, we may have changed the average durations of the single fixations made on the boundary word. Further analyses were made to check this possibility. Those revealed that for the reduced set, just as for the complete set, reading time of the boundary word was influenced by its stimulus type and word length. Both resulted in a reliable main effect $(282 \mathrm{msec}$ vs. $274 \mathrm{msec}$ vs. $\left.253 \mathrm{msec}), F_{1}(2,54)=22.15, p<.0005, F_{2}(2,696)=109.49, p<.0005\right)$ and $(259 \mathrm{msec}$ vs. $280 \mathrm{msec}), F_{1}(1,27)=26.92, p<.0005, F_{2}(2,696)=176.24, p<.0005$ without any reliable interactions.
} 


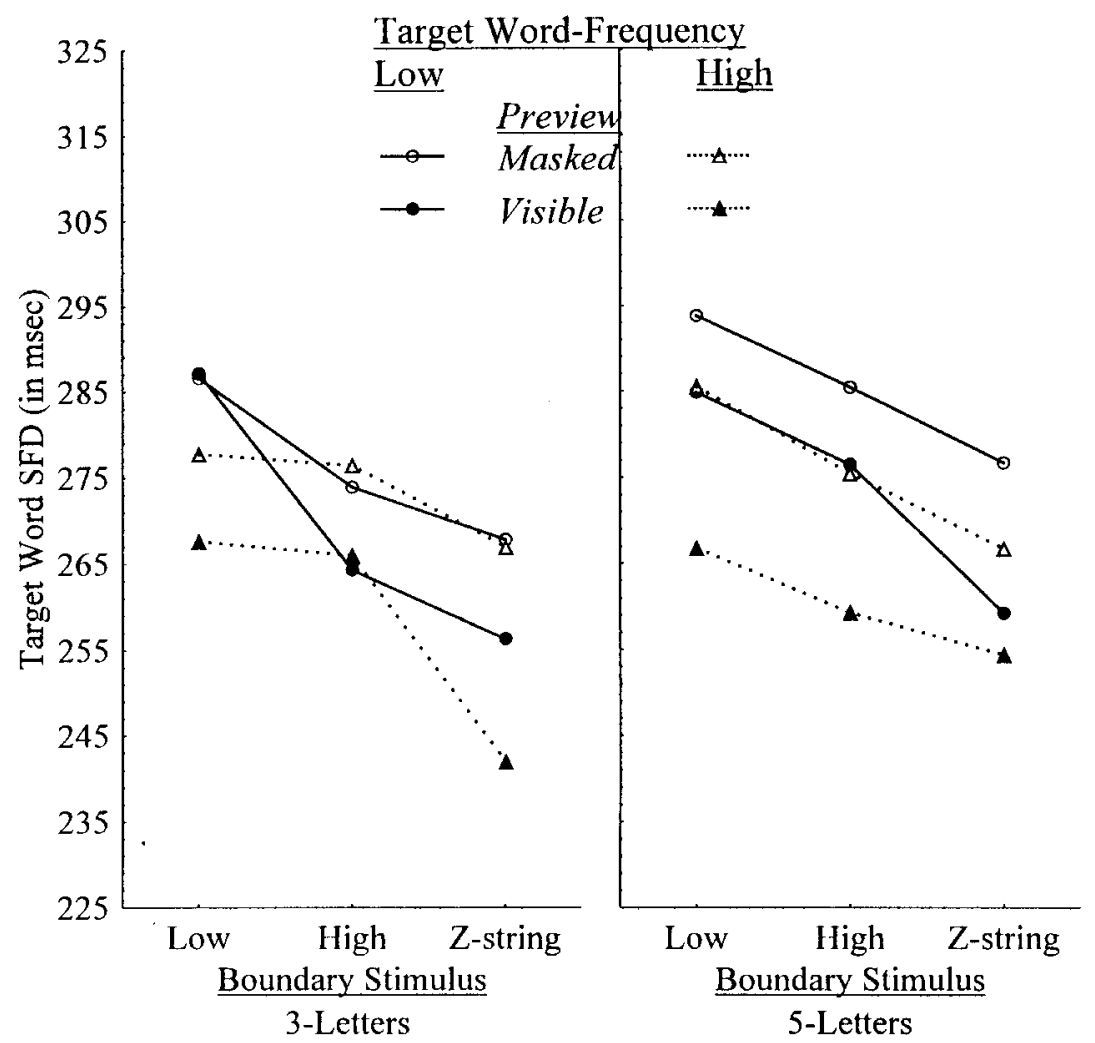

FIG. 4. Mean single-fixation durations ( $\mathrm{SFD}$, in $\mathrm{msec}$ ) on the target words as a function of their frequency and preview condition, and the type and length of the boundary stimuli.

As was found for the gaze duration measure, target word single-fixation durations (see Figure 4) showed a clear effect of the parafoveal preview condition, $F_{1}(1,27)=28.41, p<$ $.0005, F_{2}(1,696)=59.65, p<.0005$. However, the overall interaction between parafoveal preview and boundary stimulus type was at best marginal, $F_{1}(2,27)=1.76, p>.10$, $F_{2}(2,696)=1.87, p>.15$. There was no significant interaction between parafoveal preview and boundary stimulus type for any of the four conditions obtained by crossing boundary stimulus length with target word frequency, $F \mathrm{~s}<1.8$. The effect of the boundary stimulus type was significant, $F_{1}(2,54)=23.70, p<.0005, F_{2}(2,696)=36.72, p<.0005$, in both the visible, $F_{1}(2,54)=19.77, p<.0005, F_{2}(2,696)=23.70, p<.0005$, and the masked parafoveal preview condition, $F_{1}(2,54)=12.62, p<.0005, F_{2}(2,696)=10.87, p<.0005$. This again indicates a spill-over effect from the boundary word on target word processing.

\section{Target Word First-fixation durations}

For compatibility with the analyses made in earlier studies (see Henderson \& Ferreira, 1990; Kennison \& Clifton, 1995), we also analysed the mean first-fixation duration on the target words (whatever the number of consecutive fixations on this word). Results from 
this analysis are presented in Figure 5. They show a clear effect of the parafoveal preview condition, $F_{1}(1,27)=33.39, p<.0005, F_{2}(2,696)=54.30, p<.0005$, but do not reveal a decrease of the preview benefit with foveal load. Neither the boundary word frequency, nor the boundary word length, nor the target word frequency formed the basis for an overall modification of the parafoveal preview benefit. These results from the first-fixation duration measure therefore fail to replicate those of Henderson and Ferreira (1990) and Kennison and Clifton (1995).

\section{GENERAL DISCUSSION}

In recent years, several models of eye movement control in reading have proposed the idea of serial word processing. According to these serial attentional theories, the word on which the eyes fixate (the foveal word) is fully processed before attention shifts to the next word (the parafoveal word) and the system starts programming an eye movement towards this word. In one of the earliest of these models (Morrison, 1984; see also McConkie, 1979), the amount of parafoveal processing was limited to the time needed

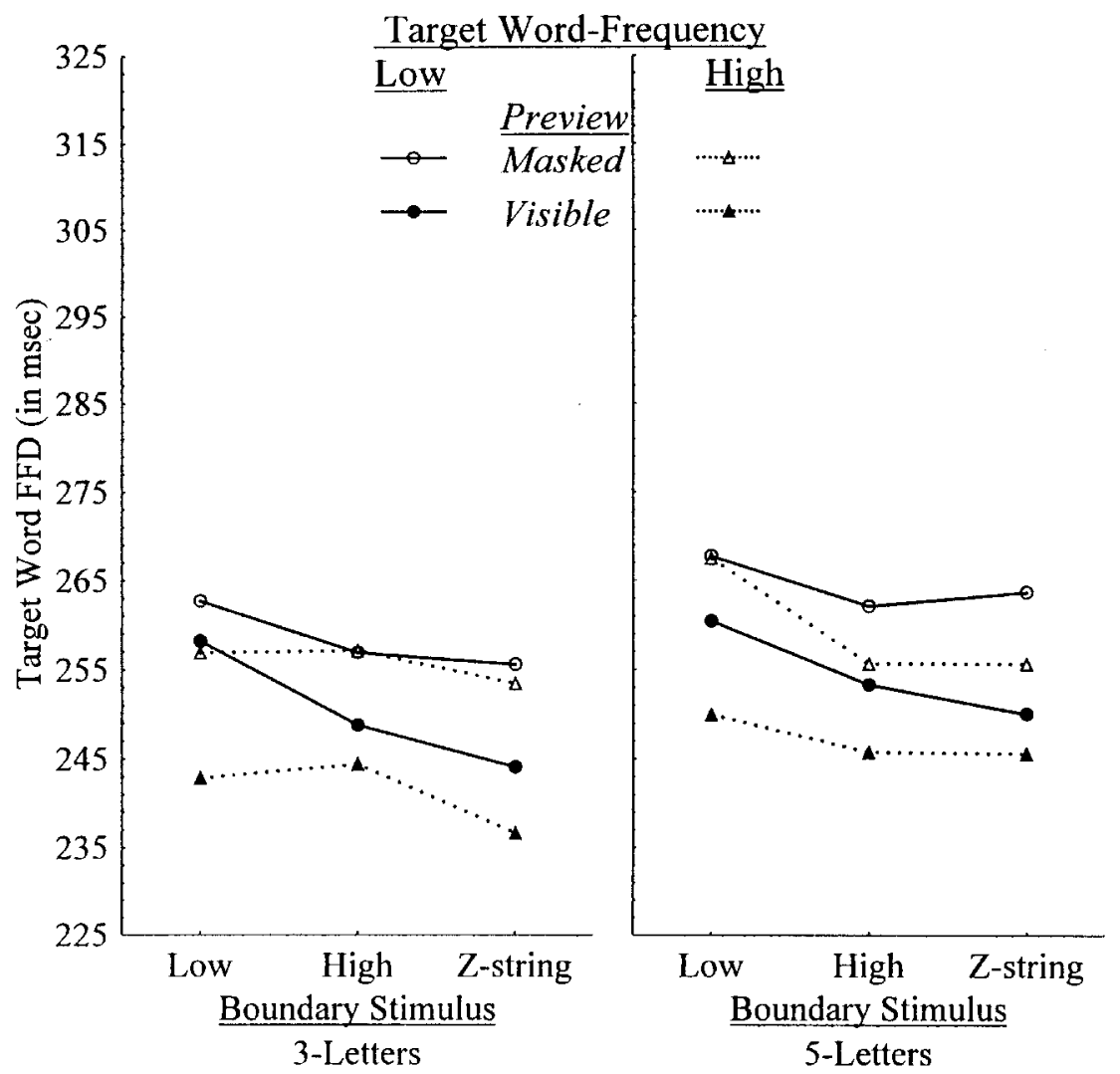

FIG. 5. Mean first-fixation durations (FFD, in $\mathrm{msec}$ ) on the target words as a function of their frequency and preview condition, and the type and length of the boundary stimuli. 
for programming a saccade to the parafoveal word and, therefore, was assumed to be rather fixed. This idea did not fit with the subsequent observation that the parafoveal preview benefit depended on the foveal load: that is, subjects profited more from seeing the next word in parafoveal vision when the foveal word was easy than when it was difficult (Henderson \& Ferreira, 1990; Kennison \& Clifton, 1995). To account for this effect of foveal load within the framework of serial attentional theory, Henderson and Ferreira proposed the eye movement programming deadline hypothesis. According to this idea, when the eyes stay too long at the same location, a refixation of the foveal word is programmed. In a number of cases, the programming of this refixation can be cancelled and adjusted to a forward saccade to the next word if in the meantime the foveal word has been recognized. On these particular occasions, the parafoveal word is processed for a shorter period of time, and preview benefit should be smaller. As the eye movement programming deadline is more likely to be exceeded in the case of difficult foveal words, this explains, for instance, why the parafoveal preview benefit is lower for low-frequency foveal words than for high-frequency foveal words. Two studies have reported this effect so far (Henderson \& Ferreira, 1990; Kennison \& Clifton, 1995), but for reasons that are unclear neither of them reported the most straightforward analysis, namely that according to the theory the effect should be found only for those cases in which the foveal word is processed in a single fixation with any refixation being cancelled.

The present experiment added a number of extra controls on the foveal word (the boundary word) to enhance the probability of getting a single fixation on this word. These included the use of short words (of three and five letters) and requiring the initial fixation position to be the centre of the word. Furthermore, trials on which nonetheless a double fixation on the boundary word occurred ( $2 \%$ of the cases) were excluded from the analyses. In general, the results remained in line with previous findings, at least when the gaze duration on the target word was used as dependent variable. The benefit of seeing the parafoveal word (henceforth the target word) was lower when the boundary word was of low frequency than when it was of high frequency or comprised a meaningless homogeneous string of z-letters. The parafoveal preview benefits for the last two conditions did not differ from one another, although there was a clear difference in processing time (see Figures 1 and 4). This is compatible with Henderson and Ferreira's (1990) oculomotor deadline hypothesis, if it is assumed that the deadline is situated somewhere between the average reading time for the low-frequency boundary words and the average reading time for the high-frequency boundary words.

In the present experiment, the effect of foveal load (or boundary stimulus type) on preview benefit found for the gaze durations did not generalize to other eye movement measures, such as target word first-fixation durations, single-fixation durations, and target word refixations (see Figures 3 and 5). To some extent, this might also be accounted for by Henderson and Ferreira's (1990) hypothesis, even though this hypothesis does not specify whether different eye movement measures can lead to different patterns. First, the probability of refixating a word might not be sensitive enough to reveal effects of the linguistic variable manipulated. As suggested by Vitu (1991), although two different words might show different processing rates, both words might or might not be refixated simply because a processing threshold has or has not been reached in either case. This could indeed account for the fact that no effect at all was observed for the refixation 
probability. Second, the first-fixation duration measure, which is based on all fixation cases (including one- and two-fixation cases), does not always provide a clear picture of the effects obtained because of its compound nature. As it is based on all fixation cases, it includes one- as well as two-fixation cases. As shown in several studies, the durations of single fixations are more sensitive to linguistic manipulations than are the durations of the first fixations of two-fixation cases (O'Regan \& Lévy-Schoen, 1987; Vitu \& O'Regan, 1995; Vitu et al., 1999). Effects might then be visible with first-fixation durations, depending on the proportion of one-fixation cases compared with that of two-fixation cases. Here, the proportion of two-fixation cases (about 16\%) was probably not large enough to allow parafoveal preview effects to emerge. Finally, the fact that single-fixation durations did not show a significant effect of the foveal load on the parafoveal preview benefit might have resulted from a diminution of the number of observations (restricted to single-fixation cases), which in turn might have decreased the power of the analyses. We think, however, that this account in terms of reduced power is rather unlikely, as the effects of parafoveal preview are still significant and comparable in size despite the reduced number of observations.

Several other findings are more difficult to reconcile with Henderson and Ferreira's (1990) theory. First, we have noted earlier that for gaze durations there was no difference in preview benefit between high-frequency boundary words and the z-letter string, whereas there was a difference between high-and low-frequency boundary words. On the basis of this observation one might argue that the saccade programming deadline proposed by Henderson and Ferreira must be somewhere between the time spent on lowand that spent on high-frequency boundary words. However, if we consider the average reading time for both three- and five-letter boundary words, then the programming deadline must lie between 255 and $265 \mathrm{msec}$ for three-letter boundary stimuli, but between 275 and $295 \mathrm{msec}$ for five-letter boundary stimuli (see Figure 1). It would therefore vary with the length of the fixated word, contrary to Henderson and Ferreira's notion of a fixed eye movement programming deadline (see Vitu \& O'Regan, 1995, for additional arguments against the notion of a fixed eye movement programming deadline). Henderson and Ferreira's theory should therefore be revised to account for the present findings.

Second, in our data, a modification of the parafoveal preview benefit due to the foveal load was clearly present in the case of low-frequency target words but did not reach significance in the case of high-frequency target words. ${ }^{3}$ This pattern of results reproduces Kennison and Clifton's (1995, Table 8) findings that the difference in preview benefit between high- and low-frequency boundary words is larger for low-frequency than for higher frequency target words. These two sets of findings are incompatible with Henderson and Ferreira's (1990) hypothesis. Indeed, although this hypothesis can predict

\footnotetext{
3 The absence of a reliable interaction between parafoveal preview and boundary stimulus types at the level of high-frequency boundary words might be due to stimulus selection. Distinct subsets of boundary words were paired with low-frequency target words but not with high-frequency target words (and vice versa; see the Appendix). However, analyses on the materials showed no reliable differences in neither the frequencies of the low-frequency boundary words nor the frequencies of the high-frequency boundary words that were paired with the respective subsets of low- or high-frequency target words, $F_{\mathrm{s}}<.001$.
} 
differential preview benefits due to parafoveal word frequency (parafoveal processing being more efficient for easy-to-process words), it cannot predict that the variations in parafoveal preview benefit with the foveal load depend on the parafoveal word frequency. Effects of the foveal load on the parafoveal preview benefit are supposed to result only from saccadic programming time constraints that are independent of the characteristics of the parafoveal word, and should depend only on when processing of the boundary word ends with respect to the eye movement programming deadline.

Third, Henderson and Ferreira's theory does not explain the spill-over effect that we obtained from the boundary word to the target word. If a forward saccade is programmed only when the foveal word has been fully processed, there is no reason to expect the reading time of the target word to be influenced by properties of the boundary word. Nevertheless, this was one of the most conspicuous findings of the present experiment (e.g. compare Figures 4 and 1) and has also been reported by Kennison and Clifton (1995, Table 5). In addition, spill-over effects have been mentioned regularly in the literature on eye movements in reading (see e.g. Balota, Pollatsek, \& Rayner, 1985; Rayner \& Duffy, 1986). It may be remarked that the pervasiveness of these spill-over effects does not agree with the idea, proposed in several theories of eye movement control in reading, that processing of a word is completely finished when the eyes leave that word (Henderson \& Ferreira, 1990; Just \& Carpenter, 1980; Morrison, 1984; Pollatsek \& Rayner, 1992; Rayner \& Pollatsek, 1989).

A final point against Henderson and Ferreira's (1990) interpretation of foveal-onparafoveal effects concerns the relation between the time spent on the boundary stimulus and the amount of parafoveal preview. In their theory, the reduced parafoveal preview benefit with low-frequency boundary words results from the greater time spent processing these foveal words. We have indeed verified that low-frequency words induce on average greater reading times than do the high-frequency words or z-letter strings (See Figure 1 and Footnote 2). However, a closer look at the actual time spent fixating the boundary stimulus reveals a substantial overlap in fixation durations on boundary low versus high frequency. Also, there is a certain number of cases in which fixation durations were shorter on low-frequency than on high-frequency boundary words. This is shown in Figure 6, which presents the distributions of single-fixation durations for the three types of boundary stimuli (collapsed across boundary word length and target word conditions). The distributions for low- and high-frequency words are quite similar, and only in a restricted number of instances does the relative frequency of fixation durations differ between the two. High-frequency boundary words resulted in more fixations lasting about $250-275 \mathrm{msec}$, and low-frequency boundary words resulted in some more fixations lasting about $225 \mathrm{msec}$ and also $375 \mathrm{msec}$ or longer.

Therefore, a better test of Henderson and Ferreira's (1990) hypothesis (and, indeed, any hypothesis that is specified in terms of on-line time constraints) is to look not at parafoveal preview benefit as a function of boundary stimuli that on average required different processing times, but at the actual duration of the fixation preceding the saccade from the boundary stimulus to the target word. For this analysis, fixation durations on the boundary stimulus were aggregated across all conditions, except for the visibility of the target word, and they were divided into five classes: less than 220, 220-240, 240-260, 260-280, and more than $280 \mathrm{msec}$. This resulted in just one empty cell, which was 


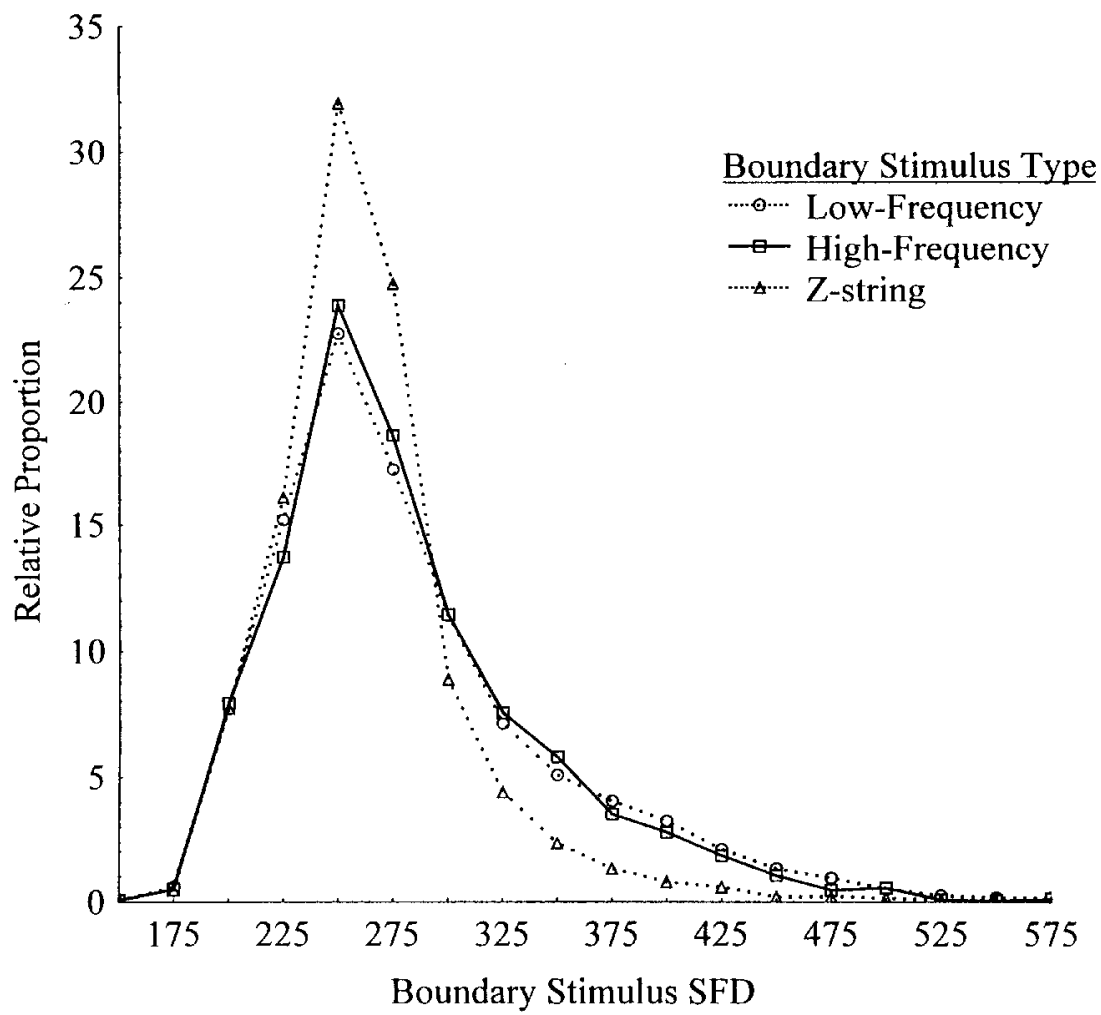

FIG. 6. Group distributions of single-fixation durations (25-msec intervals) on the different types of boundary stimuli.

replaced by the mean in that condition across all other subjects. (No items analyses were conducted because dividing the maximum of seven observations per item in to the five subclasses resulted in too many empty cells.)

According to Henderson and Ferreira (1990), we should find a drop in parafoveal preview benefit for the longer fixation durations because the eye movement programming deadline is more likely to have been exceeded. By contrast, a purely parallel processing model would predict an increase in preview benefit with longer fixation durations. Figure 7 shows the results, which are clearly in line with the parallel processing hypothesis. The difference in gaze duration between a target word that was visible and one that was masked was virtually absent for fixations below $220 \mathrm{msec}$ and grew to a difference of about $20 \mathrm{msec}$ for fixation durations above $280 \mathrm{msec}$. This interaction between singlefixation durations on the boundary word and visibility of the target word tended to be marginally reliable, $F_{1}(4,108)=2.075, p<.09$. The same effect was obtained for cases in which the target word had been processed in a single fixation, $F_{1}(4,108)=2.393, p<.06$. These results replicate earlier findings that suggested an increase of parafoveal preview benefit with longer prior fixation duration (Hogaboam, 1983; Inhoff, Topolski, \& Wang, 1992; Kerr \& McConkie, 1992; Pollatsek, Rayner, \& Balota, 1986). 


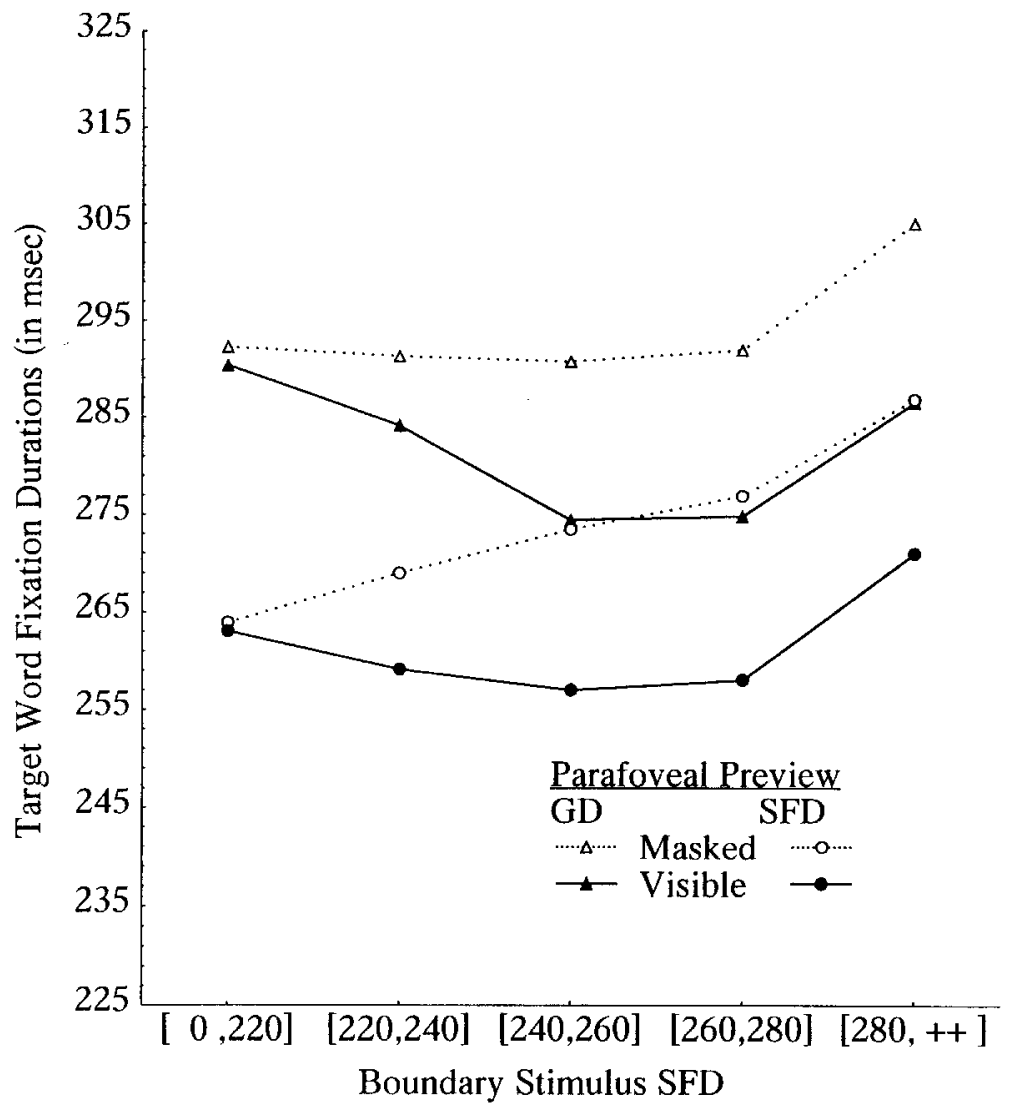

FIG. 7. Mean gaze durations (GD) and single-fixation durations (SFD, in msec) on the target words as a function of their preview condition, and $20-\mathrm{msec}$ intervals of single-fixation durations (SFD) on the boundary stimuli.

To get a clearer idea of the respective contributions of the complexity of the boundary stimulus and the actual duration of the fixation on that stimulus, we included both variables in additional analyses of target word gaze durations as a function of the visibility of the target word. To base the analysis on a reasonable number of data for each cell, the fixation durations on the boundary stimulus were divided into only two classes, which according to Figure 7 led to different preview benefits: greater or less than $240 \mathrm{msec}$.

As can be seen in Figure 8, it is clear that both fixation time and complexity of the boundary stimulus affected the parafoveal preview benefit. First, as was found in the previous analyses (see Figure 7), the preview benefit tended to be larger for long fixation durations on the boundary stimulus than for short durations, $F_{1}(1,27)=4.056, p<.06$. Second, the preview benefit tended to decrease with increased complexity of the boundary stimulus, $F_{1}(1,27)=2.497, p<.10$. The three-way interaction was not significant, $F \mathrm{~s}$ $<1$, nor was the interaction between parafoveal preview condition and boundary stimulus 


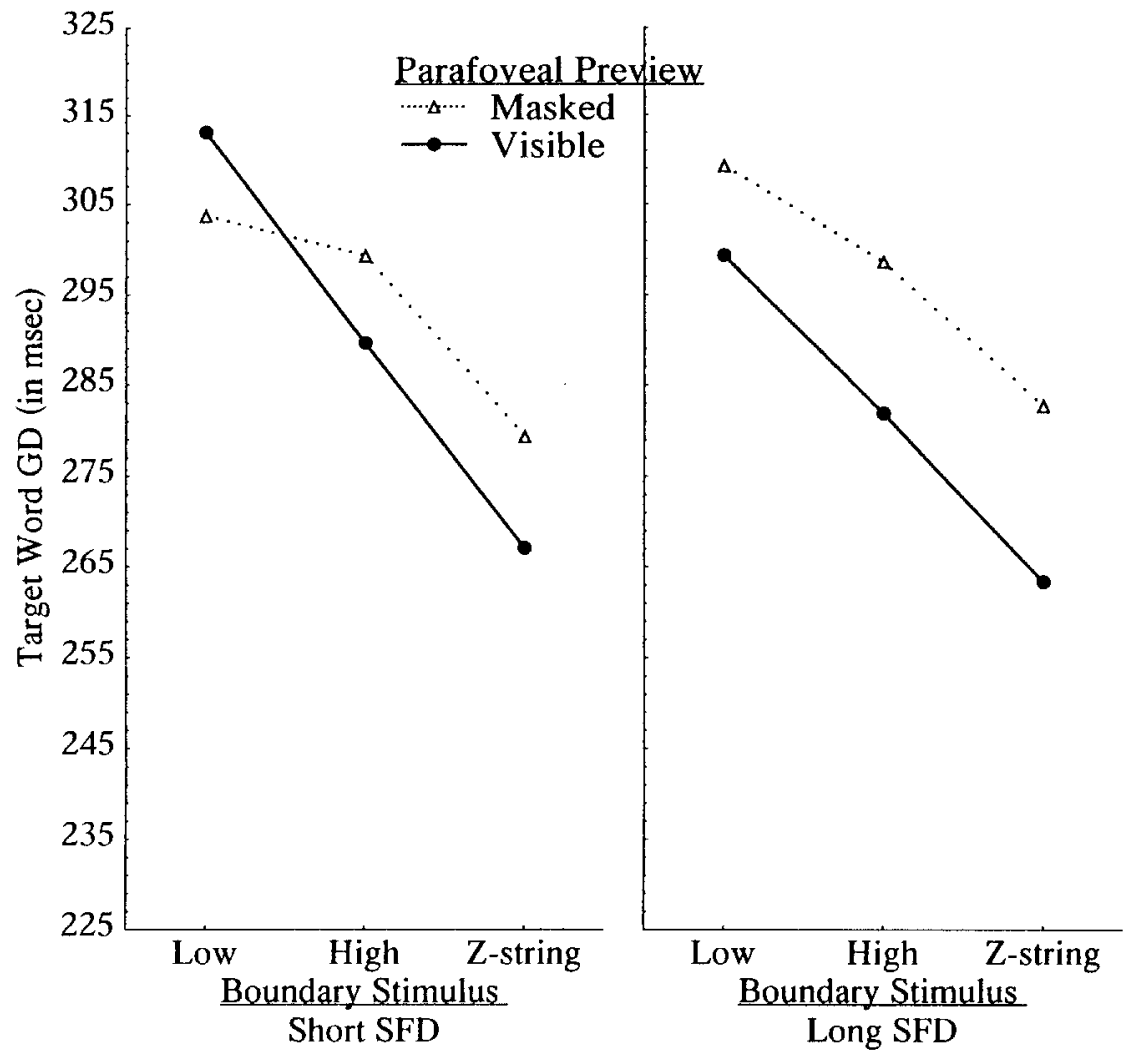

FIG. 8. Mean gaze durations (GD, in $\mathrm{msec}$ ) on the target words as a function of their preview condition and the boundary stimulus type for long or short single-fixation durations (SFD) below or above $240 \mathrm{msec}$.

type for either fixation durations shorter than $240 \mathrm{msec}$ or those longer than $240 \mathrm{msec}, F \mathrm{~s}$ $<1.1$. The effect of the type of boundary stimulus was globally significant, $F_{1}(2,54)=$ $33.86, p<.0001$.

We conclude that Henderson and Ferreira's (1990) interesting interpretation of the influence of foveal load on the parafoveal preview benefit effect within the framework of the serial model is consistent only with a restricted analysis of the data. As soon as the picture is expanded and includes, for instance, boundary stimuli of different lengths plus analyses of the distribution of fixation times, the assumption of a fixed eye movement programming deadline must be abandoned. When we analyse the data as a function of the actual time that readers spent on the boundary words, there is clear evidence for parallel processing in the interaction between foveal and parafoveal word processing. The parafoveal preview benefit increased with increasing fixation durations up to $280 \mathrm{msec}$.

At this point, it may be interesting to see how a parallel model could account for our data. In this attempt, we will rely heavily on ideas of Schiepers (1980) that have received little attention in the literature thus far. According to Schiepers, foveal and parafoveal word processing happen in parallel, but with a time delay of $90 \mathrm{msec}$ per degree of 
eccentricity. Typically, this should result in a time delay of around $200 \mathrm{msec}$ for the parafoveal word. Parafoveal word processing is most effective when it can be combined with foveal processing on the next fixation. To achieve this, the eyes cannot stay too long on the boundary word; otherwise the synchrony between parafoveal processing on fixation $n$ and foveal processing on fixation $n+1$ will be lost. This theory makes the following predictions.

First, because the parafoveal word is shifted one letter position (i.e. one third of a degree) more into the parafovea with a five-letter boundary than with a three-letter boundary word, the most effective time to move the eyes should be $30 \mathrm{msec}$ longer for a five-letter boundary stimulus. This may account for the difference in reading times as a function of the length of the boundary stimulus, even when this stimulus is made up of a homogeneous z-string.

Second, Schiepers points to the fact that a parafoveal preview benefit can be obtained only when there is a time match between the arrivals of the information from different fixations. If fixation $n$ is too long, the deadline preserving the synchrony between fixation $n$ and fixation $n+1$ has been exceeded, and the parafoveal preview benefit is decreased. This hypothesis might explain effects of the foveal processing load on parafoveal processing. As low-frequency words are generally fixated longer, the synchrony between the information extracted on fixations $n$ and $n+1$ is more likely to be lost with low-frequency boundary words than with high-frequency boundary words or letter strings. However, we have also observed that parafoveal preview is increased when the time spent on the boundary stimulus is longer, and that effects of the foveal processing load tend to be more likely after short fixation durations. This cannot be accounted for by the original hypothesis proposed by Schiepers, which predicts that preview benefits should be reduced for very long fixation durations.

A way to counter this is to assume that on some occasions there may be a tension between the time needed for processing the foveal word and the saccade execution deadline; executing a saccade before the potential synchrony between parafoveal processing in the current fixation and foveal processing in the next fixation would be lost. This may result in a number of premature saccade triggerings (and hence spillover effects), although early-triggered saccades might also be made in response to predetermined oculomotor scanning strategies (see Vitu \& O’Regan, 1995; also Morrison, 1984). In those cases, processing of the boundary stimulus is unfinished when the eyes land on the target word and will have to continue while the eyes are on the target word. As a consequence, processing of the target word might be either delayed (which decreases the likelihood that the synchrony between the information extracted on fixation $n$ and that extracted on fixation $n+1$ is preserved), or slowed down (which in turn reduces the benefit from having seen the target word in parafoveal vision on fixation $n$ ). The likelihood that the boundary word has not been completely processed at the time that the saccade is launched will depend on the duration of the fixation on the boundary word (which will also have implications for the amount of parafoveal processing that is possible) and on the difficulty of the boundary word. So, the parafoveal preview benefit would be especially reduced for low-frequency boundary words and for short fixation durations, as in the pattern we observed (see Figure 8). To some extent, one might indeed wonder whether the observed decrease in preview benefit with the foveal processing load results 
from less visual information being extracted in parafovea, or from larger interference effects on subsequent processing on word $n+1$ due to spill-over effects.

In sum, the results of the present experiment can be interpreted more easily within a parallel framework that is very similar to Schiepers' theory than within Henderson and Ferreira's (1990) serial model. According to the parallel framework, the parafoveal preview benefit is affected more by timing constraints than by the processing load of the foveal word. Parafoveal processing depends on three factors related to timing constraints: (1) the time that the word is available in parafoveal vision (which affects how much information could be extracted in parafoveal vision), (2) the time spent processing the foveal word (which affects the amount of processing that remains to be done on the foveal word after the eyes have left it or the size of spill-over effects), and (3) the extent to which the parafoveal processing on fixation $n$ can be integrated with the foveal processing on fixation $n+1$ (which depends on the time between the fixations).

Although a model within Schiepers' framework seems a good candidate to explain our findings (especially because it can account for the longer fixation durations on the fiveletter boundary words; see earlier), it should be acknowledged that there are several other parallel processing hypotheses that could also explain our data. Take, for instance, a model with parallel processing of the foveal and the parafoveal word but with limited processing capacity. In such a framework, the increase in parafoveal preview benefit with more time spent fixating the foveal word could result purely from more information being extracted in the parafovea with long fixation times. On the other hand, effects of the foveal processing load would result from the fact that more resources are needed to process a low- than a high-frequency foveal word, and therefore fewer resources are available for extraction of the parafoveal word information in the case of a low-frequency word. This would particularly apply to cases where the boundary word has been fixated for a short period of time, which might not be sufficient to extract a great amount of parafoveal information.

\section{REFERENCES}

Baayen, R.H., Piepenbrock, R., \& van Rijn, H. (1993). The CELEX lexical database [CD-ROM]. Philadelphia, P.A.: Lexical Data Consortium, University of Pennsylvania.

Balota, D.A., Pollatsek, A., \& Rayner, K. (1985). The interaction of contextual constraints and parafoveal visual information in reading. Cognitive Psychology, 17, 364-390.

Balota, D.A., \& Rayner, K. (1991). Word recognition in foveal and parafoveal vision: The range of influence of lexical variables. In D. Besner \& G. Humphreys (Eds.), Basic processes in reading: Visual mord recognition (pp. 198-232). Hillsdale, NJ: Lawrence Erlbaum Associates, Inc.

Bouma, H. (1978). Visual search and reading: Eye movements and functional visual field: A tutorial review. In J. Requin (Ed.), Attention and Performance VII (pp. 115-146). Hillsdale, NJ: Lawrence Erlbaum Associates, Inc.

Brysbaert, M., Vitu, F., \& Schroyens, W. (1996). The right visual field advantage and the optimal viewing position effect: On the relation between foveal and parafoveal word recognition. Neuropsychology, 10, 385-395.

Dunn-Rankin, P. (1978). The visual characteristics of words. Scientific American, 238, 122-130.

Henderson, J.M., \& Ferreira, F. (1990). Effects of foveal processing difficulty on the perceptual spin in reading: Implications for attention and eye movement control. Fournal of Experimental Psychology: Learning, Memory and Cognition, 16, 417-429.

Hirsch, J., \& Curcio, C.A. (1989). The spatial resolution capacity of human foveal retina. Vision Research, 29, 1095-1101. 
Hogaboam, T.W. (1983). Reading pattern in eye movement data. In K. Rayner (Ed.), Eye movements in reading: Perceptual and language processes (pp. 309-331). New York: Academic Press.

Inhoff, A.W. (1989). Parafoveal processing of words and saccade computation during eye fixations in reading. Fournal of Experimental Psychology: Human Perception and Performances, 15, 544-555.

Inhoff, A.W., \& Rayner, K. (1980). Parafoveal word perception: A case against semantic preprocessing. Perception E Psychophysics, 27, 457-464.

Inhoff, A.W., \& Rayner, K. (1986). Parafoveal word processing during eye fixations in reading: Effects of word frequency. Psychological Reviem, 87, 329-354.

Inhoff, A.W., Topolski, R., \& Wang, J. (1992). Saccade programming during short duration fixations: An examination of copytyping, letter detection, and reading. Acta Psychologica, 81, 1-21.

Just, M.A., \& Carpenter, P.A. (1980). A theory of reading: From eye fixations to comprehension. Psychological Reviem, 87, 329-354.

Kennison, S.M., \& Clifton, C.C. (1995). Determinants of parafoveal preview benefit in high and low working memory capacity readers: Implications for eye movement control. Fournal of Experimental Psychology: Learning, Memory, and Cognition, 21, 68-81.

Kerr, P.W., \& McConkie, G.W. (1992). Tomards a mathematical description of eye movements during reading. Paper presented at the XXVth International Congress of Psychology, Brussels.

McCann, R.S., Folk, C.L., \& Johnston, J.C. (1992). The role of spatial attention in visual word processing. Fournal of Experimental Psychology: Human Perception and Performance, 18, 1015-1029.

McConkie, G.W. (1979). On the role and control of eye movements in reading. In P.A. Kolers, M.E. Wrolstad, \& H. Bouma (Eds.), Processing of visible language, Vol. 1 (pp. 37-48). New York: Plenum.

McConkie, G.W., Kerr, P.W., Reddix, M.D., Zola, D., \& Jacobs, A.M. (1989). Eye movement control during reading: II. Frequency of refixating a word. Perception \& Psychophysics, 46, 245-253.

McConkie, G.W., \& Rayner, K. (1975). The span of effective stimulus during a fixation in reading. Perception E Psychophysics, 17, 578-586.

Morrison, R.E. (1984). Manipulation of stimulus onset delay in reading: Evidence for parallel programming of saccades. Fournal of Experimental Psychology: Human Perception and Performance, 10, 667-682.

Mozer, M.C., \& Behrmann, M. (1991). On the interaction of selective attention and lexical knowledge: A connectionist account of neglect dyslexia. Journal of Cognitive Neuroscience, 2, 96-123.

Nazir, T.A., O'Regan, J.K., \& Jacobs, A.M. (1991). On words and their letters. Bulletin of the Psychonomic Society, 29, 171-174.

O’Regan, J.K., \& Lévy-Schoen, A. (1987). Eye movements strategy and tactics in word recognition and reading. In M. Coltheart (Ed.), Attention and performance XII: The psychology of reading (pp. 363383). Hove, UK: Lawrence Erlbaum Associates Ltd.

O’Regan, J.K., Lévy-Schoen, A., Pynte, J., \& Brugaillère, B. (1984). Convenient fixation location within isolated words of different length and structure. Fournal of Experimental Psychology: Human Perception and Performance, 10, 250-257.

Pollatsek, A., \& Rayner, K. (1990). Eye movement guidance in reading: the role of parafoveal letter and space information. Fournal of Experimental Psychology: Human Perception and Performance, 16, 268-281.

Pollatsek, A., \& Rayner, K. (1992). Eye movement and scene perception. Special issue: Object perception and scene analysis. Canadian Fournal of Psychology, 46, 342-376.

Pollatsek, A., Rayner, K., \& Balota, D.A. (1986). Inferences about eye movement control from the perceptual span in reading. Perception ES Psychophysics, 40, 123-130.

Rayner, K. (1979). Eye guidance in reading: Fixation locations within words. Perception, 8, 21-30.

Rayner, K., \& Duffy, S.A. (1986). Lexical complexity and fixation times in reading: Effects of word frequency, verb complexity, and lexical ambiguity. Memory E Cognition, 14, 191-201.

Rayner, K., McConkie, G.W., \& Zola, D. (1980). Integrating information across eye movements. Cognitive Psychology, 12, 206-226.

Rayner, K., \& Morrison, R.E. (1981). Eye movements and identifying words in parafoveal vision. Bulletin of the Psychonomic Society, 17, 135-138.

Rayner, K., \& Pollatsek, A. (1989). The psychology of reading. Englewood Cliffs, NJ: Prentice-Hall.

Rayner, K., Sereno, S.C., \& Raney, G.E. (1996). Eye movement control in reading: A comparison of two types of models. Fournal of Experimental Psychology: Human Perception and Performance, 22, 1188-1200. 
Rayner, K., Well, A.D., Pollatsek, A., \& Bartera, J.H. (1982). The availability of useful information to the right of fixation in reading. Perception $\&$ Psychophysics, 31, 537-550.

Schiepers, C.W. (1980). Response latency and accuracy in visual word recognition. Perception $E^{-}$ Psychophysics, 27, 71-81.

Vitu, F. (1990). Le guidage oculaire pendant la lecture. Unpublished doctoral dissertation, University of Paris V, Paris.

Vitu, F. (1991). The influence of parafoveal preprocessing and linguistic context on the optimal landing position effect. Perception ES Psychophysics, 50, 58-75.

Vitu, F., McConkie, G.W., Kerr, P.W., \& O’Regan, J.K. (1999). Within-mord eye behavior during reading: Effects of initial fixation location on the gaze duration and its components. Manuscript in preparation.

Vitu, F., \& O'Regan, J.K. (1995). A challenge to current theories of eye movements in reading. In J.M. Findlay, R. Walker, \& R.W. Kentridge (Eds.), Eye movement research: Mechanisms, processes and applications (pp. 381-392). Amsterdam: North-Holland.

Vitu, F., O'Regan, J.K., Inhoff, A.W., \& Topolski, R. (1995). Mindless reading: Eye movement characteristics are similar in scanning letter strings and reading texts. Perception $E_{S}$ Psychophysics, $57,352-364$.

Vitu, F., O'Regan, J.K., \& Mittau, M. (1990). Optimal landing position in reading isolated words and continuous text. Perception \& Psychophysics, 47, 583-600.

Original manuscript received 19 December 1996 Accepted revision received 21 October 1998

\section{APPENDIX}

This appendix contains the means and standard deviations $(S D)$ of the word frequencies in the Dutch CELEX data base (Baayen, Piepenbrock, \& van Rijn, 1993) for the subsets of boundary stimuli and target words used in the experiment. Four counterbalancing lists were constructed by crossing the subsets labelled a and $b$ (presenting the target words masked or visible in parafoveal vision), and by crossing the subsets $\mathrm{c}$ and $\mathrm{d}$ (pairing the target words with the three- or five-letter boundary stimuli, given a particular boundary stimulus type).

\begin{tabular}{|c|c|c|c|c|c|c|}
\hline & \multicolumn{2}{|c|}{ Boundary Words } & & & \multicolumn{2}{|c|}{ Target Words } \\
\hline & Mean & $S D$ & & & Mean & $S D$ \\
\hline Frequency & & & Frequency & Subsets & & \\
\hline \multicolumn{7}{|l|}{ Three-letter } \\
\hline \multirow[t]{4}{*}{ Low } & .90 & .41 & Low & $\mathrm{a}, \mathrm{c}$ & .64 & .19 \\
\hline & .90 & .41 & & $\mathrm{~b}, \mathrm{c}$ & .64 & .17 \\
\hline & .92 & .41 & High & $\mathrm{a}, \mathrm{c}$ & 69.13 & 44.76 \\
\hline & .92 & .41 & & $\mathrm{~b}, \mathrm{c}$ & 69.82 & 45.25 \\
\hline \multirow[t]{4}{*}{ High } & 64.43 & 45.24 & Low & $\mathrm{a}, \mathrm{c}$ & .65 & .18 \\
\hline & 64.39 & 46.71 & & $\mathrm{~b}, \mathrm{c}$ & .65 & .17 \\
\hline & 64.53 & 50.74 & High & $\mathrm{a}, \mathrm{c}$ & 69.53 & 46.15 \\
\hline & 64.66 & 42.51 & & $\mathrm{~b}, \mathrm{c}$ & 69.04 & 45.60 \\
\hline \multirow[t]{4}{*}{$\mathrm{z}$-string } & - & - & Low & $\mathrm{a}, \mathrm{c}$ & .64 & .17 \\
\hline & - & - & & $\mathrm{b}, \mathrm{c}$ & .64 & .16 \\
\hline & - & - & High & $\mathrm{a}, \mathrm{c}$ & 68.91 & 44.40 \\
\hline & - & - & & $\mathrm{b}, \mathrm{c}$ & 68.93 & 48.99 \\
\hline
\end{tabular}


1046 SCHROYENS ET AL.

\begin{tabular}{lrrlrrr}
$\begin{array}{c}\text { Five-letter } \\
\text { Low }\end{array}$ & .72 & .27 & Low & a,d & .65 & .17 \\
& .72 & .27 & & b,d & .65 & .17 \\
& .71 & .27 & High & a,d & 68.91 & 45.92 \\
High & .71 & .27 & & b,d & 68.93 & 46.10 \\
& 108.52 & 47.49 & Low & a,d & .64 & .18 \\
& 108.94 & 48.56 & & b,d & .64 & .17 \\
& 108.77 & 47.96 & High & a,d & 69.38 & 44.96 \\
\multirow{2}{*}{ z-string } & 108.69 & 48.18 & & b,d & 68.98 & 46.84 \\
& - & - & Low & a,d & .64 & .18 \\
& - & - & & b,d & .64 & .18 \\
& - & - & High & a,d & 68.28 & 42.77 \\
& - & - & & b,d & 70.46 & 53.80 \\
\hline
\end{tabular}

